(2) Open Access Full Text Article

REVIEW

\title{
Post-marketing access to orphan drugs: a critical analysis of health technology assessment and reimbursement decision-making considerations
}

This article was published in the following Dove Press journal:

Orphan Drugs: Research and Reviews

9 January 2014

Number of times this article has been viewed

\author{
Georgi Iskrov \\ Rumen Stefanov \\ Department of Social Medicine \\ and Public Health, Medical University \\ of Plovdiv, Plovdiv, Bulgaria
}

\begin{abstract}
This study aims to explore the current rationale of post-marketing access to orphan drugs. As access to orphan medicinal products depends on assessment and appraisal by health authorities, this article is focused on health technology assessment (HTA) and reimbursement decision-making considerations for orphan drugs. A critical analysis may identify important factors that could predetermine the combined outcomes of these two processes. Following this objective, an analytical framework was developed, comprising three overlaying issues: to outline what is currently done and what needs to be done in the field of HTA of orphan drugs, to synthesize important variables relevant to the reimbursement decision-making about orphan drugs, and to unveil relationships between theory and practice. Methods for economic evaluation, cost-effectiveness threshold, budget impact, uncertainty of evidence, criteria in reimbursement decision-making, and HTA research agenda are all explored and discussed from an orphan drug perspective. Reimbursement decision-making for orphan drugs is a debate of policy priorities, health system specifics, and societal attitudes. Health authorities need to pursue a multidisciplinary analysis on a range of criteria, ensuring an explicit understanding of the trade-offs for decisions related to eligibility for reimbursement. The only reasonable way to accept a higher valuation of orphan drug benefits is if these are demonstrated empirically. Rarity means that the quality of orphan drug evidence is not the same as for conventional therapies. Closing this gap is another crucial point for the timely access to these products. The generation of evidence goes far beyond pre-market authorization trials and requires transnational cooperation and coordination. Early constructive dialogue among orphan drug stakeholders and elaboration of orphan drug-tailored methodology tools could set the scene for ongoing accumulation of evidence, as well as for proper and timely assessment and appraisal.
\end{abstract}

Keywords: orphan drugs, health technology assessment, reimbursement, decision-making

\section{Introduction}

\section{Economic rationale of orphan designation}

By analyzing the economic rationale of orphan drug designation, it is not difficult to determine the epidemiological nature of this otherwise purely economic conception. Rare disease therapies would be highly unattractive under standard market conditions from an investor perspective. The small number of patients cannot financially justify expending resources into orphan therapy research and development, which is a long, expensive, and risky process.

The most obvious impact of this rarity factor is on the economics of orphan drugs. As the population of patients with a specific rare disease is very small, the investments must be recouped by increasing the price of the product. However, rarity plays another 
important role: it is much more difficult to produce good quality evidence for orphan drugs as the fewer number of afflicted patients makes it consistently harder to conduct clinical trials for rare diseases. Furthermore, the epidemiology of this group of disorders is still poorly explored, which is why the prognosis for long-term benefits of orphan therapies relies almost exclusively on surrogate indicators, making the final clinical outcomes very speculative. This assumption adds considerable uncertainty to the reimbursement decisionmaking process of orphan drugs.

It has been acknowledged that, while regulatory incentives have stimulated research and development of orphan therapies on a global level, equitable and timely access to approved orphan medicinal products for rare disease patients remains an issue. ${ }^{1}$ Furthermore, effective market access and utilization varies greatly between and within national jurisdictions. ${ }^{2}$ Moreover, it is not an unusual situation to have almost contradictory reimbursement recommendations about the same orphan medicinal product in different countries. ${ }^{3}$

\section{Assessment of orphan drug value}

Access to orphan drugs at a national level is hindered by the limited evidence, expertise, and experience on rare diseases. Highlighted bottlenecks include lack of clinical and epidemiological data, inappropriate pricing and reimbursement procedures, and absence of public awareness of these problems. ${ }^{4}$ Nevertheless, it must be underlined that universal access to orphan drugs is not a panacea. Keeping in mind the restricted availability of resources, it is not a question whether to prioritize rarity, but to create legitimate mechanisms for properly assessing orphan drug value and using this value in an optimal way according to societal views and needs. ${ }^{4}$ The concept of health technology assessment (HTA) has been circulating for a while, but the recent increased pressure on public health spending is the reason for the growing interest in HTA integration into the reimbursement decision-making process. $^{5}$

In response, governments are currently looking for tools that can support the use of safe and effective health technologies and also that impose order in health care costs at the same time. HTA and its multispectral inventory is probably the key to create a sustainable environment for timely and adequate access to orphan drugs. Given the fact that both orphan drugs and HTA are relatively new public health topics, there is a number of questions about their optimal interaction. Social relevance, cost-effectiveness, and rarity are just some of these parameters, whose exact role and influence in the decision-making process need to be thoroughly studied and described.

\section{Objective}

This study aimed to explore the current rationale of postmarketing access to orphan drugs. As access to orphan medicinal products depends on 1) assessment, and 2) appraisal by health authorities, this article is focused on HTA and reimbursement decision-making considerations for orphan drugs. A critical analysis may identify important factors that could predetermine the combined outcomes of these two processes.

\section{Materials and methods}

Following this objective, an analytical framework was developed, comprising three overlaying issues: 1) to outline what is done and what needs to be done in the field of HTA of orphan drugs; 2) to synthesize important variables relevant to the reimbursement decision-making about orphan drugs; and 3 ) to unveil relationships between theory and practice.

As both HTA and reimbursement decision-making are very extensive fields, several key features to be reviewed and considered in the critical analysis are predefined. Methods for economic evaluation, cost-effectiveness threshold, budget impact, uncertainty of evidence, criteria in reimbursement decision-making, and HTA research agenda were all explored and discussed from an orphan drug perspective. Available biomedical literature, as well as publicly disclosed regulatory documents and position papers are reviewed to analyze the use of HTA concepts and tools in orphan drug cases, as well as their application in reimbursement decision-making were reviewed.

For the purposes of this study, no distinction was made between the different legal definitions of orphan drugs across jurisdictions. This was justified, as orphan drugs are exclusively regarded as medicinal products that are intended for the diagnosis, prevention, or treatment of a life-threatening or chronically debilitating condition affecting a very small number of patients. The difference here comes only from the officially accepted rare disease prevalence, which varies between the EU, USA, Japan, and Australia. The study framework was basically organized under EU settings; however, it was not geographically limited and relevant papers from outside the EU were considered as well.

In some cases, similar studies, focused on oncological medicines for rare tumors, were accepted as eligible for critical analysis, despite the fact that these were not officially orphan designated products. This is motivated by the clear set 
of analogies between these two groups of health technologies: 1) they were both considered innovative therapies; 2) both new oncological and orphan drugs have a high acquisition cost; and 3) evidence of clinical effectiveness in both cases usually relies on surrogate points and extrapolation.

\section{Results and discussion \\ Methods for economic evaluation of orphan drugs}

The inventory of methods for economic evaluation of health technologies has recently evolved enormously. The choice of evaluation method is essential and could significantly affect subsequent reimbursement decision-making. The conduct of such assessment must follow certain principles of quality and comparability of outcomes. ${ }^{6}$ All relevant costs and outcomes of the reviewed health technology were properly identified and measured. Regarding outcomes, they included both positive and negative ones, as well as the added value for society. It is important to clarify whether they were surrogate or endpoints. Intermediate outcomes usually have less value for decision-makers as they are mostly predictive and often cannot provide adequate projection of long-term outcomes. Nevertheless, the application of surrogate endpoints in rare diseases and orphan drugs should not be totally rejected, especially in the process of developing and approving new therapies.

Uncertainty for surrogates will always exist in rare diseases with limited clinical data. Miyamoto and Kakkis have advocated that established criteria and precise guidance to support researchers and investors would help foster the effective use of appropriate surrogates and improve access to marketing authorization. ${ }^{7}$

The choice of comparator largely predetermined the results of this analysis. Economic evaluation represents a comparative analysis of at least two competing health technologies (eg, innovative versus conventional) with respect to costs and outcomes. The latter are usually defined in terms of health gain, using generic measures of health outcome (eg, quality-adjusted life year [QALY]) or clinical indicators. When a new product belongs to a well-known therapeutic category, its efficacy is compared to similar drugs from the same category. However, orphan drugs almost always represent a new therapeutic group themselves. In this context, the appraisal could be possibly more implicit. Innovative therapies are often the very first response to unmet health needs, which is an important societal factor in decision-making. ${ }^{8}$

Regarding orphan drugs, the cost-utility analysis (CUA) is probably the most adequate method of economic evaluation as it allows comparisons between technologies, using weighted values for quality of life. This feature partially solves the problem of comparator selection, because other methods (eg, cost-effectiveness analysis [CEA]) focus more on the specific clinical outcomes, measured in physical units. That is why CUA is the recommended method when it comes to cases where quality of life is expressly stated as the most important indicator for assessment (eg, life support therapies with serious side effects or therapies that affect morbidity but not mortality). ${ }^{6}$ On the other hand, the use of CUA may be restricted because of the lack of agreement on the instruments to measure and evaluate quality of life indicators in different patient populations, as well as on the transformation of patient preferences into quantitative units. ${ }^{6}$

QALYs are the predominantly used quality of life indicator in reimbursement decision-making. This methodology, as currently constituted, focuses on health as opposed to well-being, more generally. ${ }^{9}$ Nevertheless, health status is only one of the components of well-being. HTA and reimbursement decision-making need to consider the impact of health technologies and orphan drugs in particular on other health and social policies. Additionally, QALYs have a different potential when used at different levels of the health system. As decisions are most often applied to the whole population, QALYs are supposed to give priority to the common public perspective and not to one of the patients who will be directly affected by a specific health technology. ${ }^{10}$ From the orphan drug point of view, the majority of the general population have no experience with rare diseases. Therefore, would society be able to appraise such a specific issue as orphan drug value? Decision-making about orphan drug reimbursement should reflect and include the views of the directly affected individuals. If orphan drugs are able to be proven cost-effective, even in a very small population, then decision-makers need to create conditions for access to these therapies. ${ }^{11}$

\section{Cost-effectiveness threshold for orphan drugs}

The use of CEA in HTA usually involves an assessment of the incremental cost-effectiveness of the new therapy compared to the existing treatments for the disease in question. ${ }^{12}$ The incremental cost-effectiveness ratio (ICER) has been regarded as a benchmark for reimbursement decision-making. ${ }^{13}$ It is clear that orphan therapies cannot meet the standard requirements for cost-effectiveness using ICER. This problem is not because of the methodology itself, but instead it is based on orphan drug specifics - high price and incertitude about the 
outcomes. The issue here is whether these ICER thresholds, which are used for other health technologies, can be directly applied in orphan drug cases.

The concept of ICER is not new. ${ }^{13}$ However, despite strong political and societal commitment for more structured rationale in reimbursement decision-making, there are very few examples for official adoption of an explicit ICER threshold. The National Institute for Health and Care Excellence (NICE) in the UK, which is the flag bearer in HTA field, has been consistently pointed out for introducing and using, albeit indirectly, ICER thresholds. ${ }^{14} \mathrm{McCabe}$ et al have cited NICE's 2004 Methods Guide, that states:

Although the use of a threshold is inappropriate, comparisons of the most plausible ICER of a particular technology compared with other programmes that are currently funded are possible and are a legitimate reference for the Committee. ${ }^{14}$

Eichler et al have provided a detailed overview of the development of and debate on ICER thresholds. ICER offers a range of theoretical advantages, including reduced burden of responsibility upon decision-makers, consistency and transparency of the decision-making process, equity, efficiency, and public trust. ${ }^{13}$ Nevertheless, it is both a politically and morally sensitive issue. Application of an explicit ICER requires comparisons and priority rankings under strict conditions that are, however, not always present in practice. Reality shows that there is no such thing as constant, contextindependent willingness-to-pay for any QALY unit gained. ${ }^{10}$ Decision-makers and society tend to give different priority to different health technologies. Not using explicit thresholds allows more room for ad hoc considerations, which may be more attractive to decision-makers. ${ }^{13}$

McCabe at al have offered further valuable comments on the factors that are taken into account when a health technology's ICER is above the conventional threshold. In this case, the Appraisal Committee considers: 1) whether the characteristics of the condition or population receiving the treatment would lead them to value the health gain produced by the intervention more highly than the estimate made in the analysis; 2) whether innovative characteristics of the intervention are such as to require to give due weight to innovativeness; and 3) whether other benefits to society are such that it is socially desirable for the treatment to be made available. ${ }^{14}$

Obviously ICER and QALYs are not the only factors in reimbursement decision-making. There are also equity, solidarity, social justice, and so on; however, it is not clear how all these are interacting in this process. Instead, ICER shows the cost-effectiveness of a health technology; it is not a measure of social justice. As a component of it, QALYs play a major role in decision-making. However, QALY methodology is criticized for not being able to reflect personalized issues. ${ }^{10}$ Different individuals may have different capacities to take advantage of longevity and quality of life. Social preferences for allocation of health care resources may also vary from the QALY-based ones.

Could rarity be a modifying factor in shaping social preferences? From a health economics perspective there are different opinions on this question. Some authors believe that rarity cannot be an argument for making orphan drugs a subject to special treatment when making reimbursement decisions. ${ }^{14}$ Others take a less firm position, arguing that cost-effectiveness should be regarded together with the social value of health technologies. ${ }^{15}$ Drummond has pointed out a problem in the possible divergence between orphan drugs' social value and the public demand for efficiency in health care expenditure. ${ }^{15}$ Orphan drugs are intended to treat serious, life-threatening or chronically debilitating conditions, where other therapies are often not available. These health technologies are expensive on an individual patient basis, but are supposed to have limited impact on the health budget as a whole, since patients with a specific rare disease are very few.

Economic evaluation and HTA in general do not make a decision whether a health technology would be publicly funded for use. They are only a tool for health authorities, whose decisions are a combination of many factors. Uncertainty of evidence is what makes regulators extremely cautious when appraising orphan drugs. The lack of accepted and established criteria for social justice and the dominance of cost-effectiveness indicators may lead to the marginalization of other factors in the decision-making process. ${ }^{10}$

\section{Budget impact of orphan drugs}

There is growing recognition that a comprehensive economic assessment of a new health technology at the time of launch requires both an economic evaluation and a budget impact analysis (BIA). ${ }^{16}$ Opportunity costs (or estimation of missed profits) is one of the reasons to apply BIA. While economic evaluation allows decision-makers to assess the effectiveness of health technologies, BIA concentrates on the impact of the adoption and use of a technology in a particular jurisdiction. Information from economic evaluations provides the basis for a positive decision for reimbursement, but BIA eventually defines what resources will be necessary to implement this decision. ${ }^{17}$ 
Some health economists blame budgetary considerations for undermining the rational application of cost-effectiveness criteria and leading to suboptimal allocation of resources for health. ${ }^{17}$ In the case of orphan drugs, budget impact is presumed to be a major issue. Health authorities attach great importance to all considerations concerning the sustainability of the health system. They are afraid that the opportunity cost of orphan drug adoption may be too high and would imply substantial changes in other health technology reimbursements by public funds. Mauskopf et al have confirmed in several studies that it is much more likely for a health technology with a significant budgetary impact to be rejected for reimbursement or subjected to additional conditions and restrictions. ${ }^{18,19}$

Another problem of orphan drug BIA is the fragmented use and interpretation of evidence. Analyzing the work of Belgian regulators, Denis et al have found that BIA of orphan drugs are very simplistic and do not always account for the full impact of these therapies. ${ }^{20}$ For example, health care costs are usually divided into several budgets and decisions are taken for each budget separately, without considering the overall impact of a health technology on the total budget at the end. This is an obstacle to the pursuit of objective economic evaluation as health technologies may have an impact on many different budgets. For example, although the reimbursement of orphan drugs would lead to increased costs for medicinal products, it may also imply a cost reduction for other health and social services.

With all this in mind, it is easy to understand governmental concerns at the national level about the cost of orphan drugs. Nevertheless, putting aside the initial fear of a significant fiscal burden, the literature shows a different picture about the budgetary impact of orphan drugs in Europe. The very first analysis of orphan drugs' budget impact was made in 2004 at a request of the European Commission. ${ }^{21}$ Researchers studied the level of prices and reimbursement in a number of EU member states. They reported orphan drugs' budgetary impact to be $0.7 \%-1.0 \%$ of total national budgets earmarked for medicines. At that time this share was forecast to reach $6 \%-8 \%$ by 2010 . Of course, this first study came only a few years after the launch of EU orphan drug legislation, and the data for analysis was scarce. However, all subsequent reviews have confirmed these initial estimates. ${ }^{22}$

The most recent and comprehensive study on this issue has generated estimates for the Eurozone and the UK for 2010-2020. ${ }^{22}$ The model constructed by Schey et al has predicted the annual per patient cost of orphan drugs to vary between $€ 1,251$ and $€ 407,631$, with the median cost being $€ 32,242$ per year. In sensitivity analyses, a peak-year orphan drug budget impact ranges between 3\%-6.6\%. The share of the total pharmaceutical market represented by orphan drugs is predicted to increase from $3.3 \%$ in 2010 to a peak of $4.6 \%$ in 2016 after which it is expected to level off through 2020, as growth falls into line with that of the wider pharmaceutical market. Schey et al have explained the steady growth of orphan drug budget impact over the next decade by the combined effect of the loss of marketing exclusivity and patent protection, the low "success rate" for drugs that have been granted orphan designation, and the diminishing penetration rate of new drugs into prevalent patient populations after a drug is first licensed. ${ }^{22}$

Uncertainty is another important reason for the inclusion of BIA when making decisions about allocating resources. When launching a new orphan drug on the market, it is unlikely to know the precise extent to which this product will be used. It is also difficult to assess the exact size of the patient population that will benefit from it. In addition, new indications for the product may be labeled later on. Uncertainty is a particularly acute problem, as decisions for reimbursement may lead to serious budget overspending. This is why regulators avoid making firm reimbursement decision about health technologies with large budgetary impacts, as the level of uncertainty may be too high to be accepted. ${ }^{17}$

\section{Uncertainty of orphan drug evidence}

Orphan drugs represent a problem for reimbursement decision-making, as they rarely meet the criteria of costeffectiveness and evidence quality. Health authorities increasingly require real world evidence on which to base their recommendations. This practice shows the importance of evidence that goes far beyond the information collected during randomized controlled trials (RCTs) and required for marketing approval. ${ }^{3}$ It is well known that RCTs are a golden standard for providing solid evidence of efficacy, but it should not be forgotten that these data are obtained from selected populations in carefully controlled conditions. Before making a reimbursement decision, health officials want to be sure that the health technology will work effectively under real world conditions. This is particularly true in cases when ICER exceeds conventional thresholds or the budgetary impact is excessive; regulators would demand higher levels of certainty in order to issue a positive recommendation.

For a number of well-known reasons, the level of clinical evidence for orphan drugs is usually low, especially in the period shortly after the marketing authorization, and orphan drug evidence is often undermined by the small number of patients in the clinical trials. Bridging this evidence gap is 
crucial for positive reimbursement recommendations. Global regulatory agencies such as the European Medicines Agency (EMA) traditionally base their drug approval decisions on assessment of quality, safety, and efficacy. The payers, however, require further evidence of benefits of the new drug over the therapeutic alternatives. ${ }^{3}$

Dupont and Van Wilder have analyzed the reimbursement decisions for orphan drugs in Belgium and France between 2002 and 2007, exploring the relationship between evidence uncertainty and health authorities' final decision. ${ }^{3}$ In Belgium, the government has approved for reimbursement a total of $22(88 \%)$ orphan drug applications. In comparison, the number of accepted reimbursement applications of nonorphan medicines, claiming additional therapeutic value (ATV), is only 74 (63\%). The results in France have been similar (96\% versus $86 \%$ ), making the authors conclude that the orphan designation may be a strong predictor for reimbursement despite poor quality of clinical evidence. For example, the authors have found that in a random control sample of 25 ATV submissions, 21 included results of at least one RCT. In the case of orphan drugs, only 13 of 25 files have included an RCT. The number of trial patients has been problematic in almost all orphan applications; ten files have included less than 100 patients each, and 16 included less than 250 patients.

The situation described above is very typical for orphan drugs. There is a need to find a compromise between adequate access to orphan drugs and regulators' concerns about quality, efficacy, and safety. Managing evidence uncertainty about orphan drugs is far more difficult because of the small number of patients and scarce expertise and experience of health care professionals. More flexible criteria should be used to assess the clinical added value of orphan drugs, as firm restrictions could negatively affect the rights of patients afflicted by rare diseases to treatment. There is a need for more quality clinical evidence for orphan drugs, so regulators and payers can more consistently appraise orphan drug risks and benefits. This issue is very important, as the progress of genomics suggests that new drugs should be expected to be more and more personalized, increasing the number of medicinal products that are subject to orphan designation. ${ }^{23}$

\section{Interaction of regulatory criteria in orphan drug reimbursement decision-making}

To ensure sustainability and credibility of the reimbursement decision-making process, policy makers have created specialized bodies to perform HTA. Despite these efforts, there are legitimate concerns among medical professionals, patients, and industry that access to innovative therapies could be greatly delayed. However, HTA is only a tool; priorities are set and decisions are made on the basis of a combination of multiple factors. In the case of orphan drugs, priority setting involves complex value-laden choices that are often ethically controversial. This controversy arises, in part, because it involves conflicting moral obligations (eg, beneficence versus distributive justice), which results in different levels of funding and opposing interests of a number of involved stakeholders. $^{24}$

Reimbursement decision-making is a question of priority setting. This crucial process is very often done ad hoc. ${ }^{25}$ In recent decades, a number of approaches to priority setting have been developed with evidence-based medicine being the most prominent. However, all these conceptions offer limited guidance to policy makers, mainly because they have been developed in isolation from each other. They concentrate on a single criterion for priority setting and do not advise on how to integrate or judge the relative importance of each criterion. ${ }^{25}$ Most frequently, regulators try to combine costeffectiveness with other considerations. These settings lack methodological rigor and appropriateness, which negatively affect their transparency and objectiveness in the end. This is why there is a need for a rational and clear approach to priority setting that guides policy makers in their choice of health interventions and that maximizes social welfare. Formalization of the priority setting process is perceived as fair and legitimate, leading to a balance and agreement among different stakeholders' interests. Any coverage limitations are most acceptable when applied in a transparent and consistent manner, which recognizes public health priorities and fiscal constraints, but also respects the individual health care right of each patient. ${ }^{26}$

It is important to reveal the direct and indirect criteria that play a role in the process of priority setting and reimbursement decision-making for orphan drugs. Rosenberg et al have examined these questions in three countries with respect to two orphan drugs. ${ }^{24}$ The main finding has been that the three different priority setting committees, working in three different health systems, have reported using essentially the same values when making reimbursement recommendations for orphan drugs: evidence, rule of rescue, and equity. Authors have confirmed that regulators place a high value on clinical evidence. A particular concern among regulators is the lack of good clinical evidence of the orphan drug's cost-effectiveness. ${ }^{24}$ It is well recognized and admitted that the application of 
standard cost-effectiveness criteria for orphan drugs is very problematic. This is much more due to the deficit of quality evidence upon which to make a proper judgment, than to the high cost. Similar priority settings have been reported by Koopmanschap et al. ${ }^{27}$ Dutch health authority representatives have identified disease severity, individual health gains, and budget impact as some of the leading factors in reimbursement decision-making. An interesting fact is that regulators have preferred health gains that include quality of life improvements over extension of life without improved quality of life. ${ }^{27}$

Rocchi et al have performed a comprehensive analysis on the particular role of economic evidence in Canadian oncology reimbursement decision-making. ${ }^{26}$ Rationales for priority setting could change, especially as treatment costs increase, and they involve clusters of factors and not simple trade-offs. Because economic evidence is based on clinical evidence, any clinical shortcomings have a direct influence on the quality and impact of the economic evidence. Authors, however, have detected some apparent inconsistencies in the evaluation of economic evidence in Canada (eg, incoherencies in ICER's eligibility for innovative medicines, different use of surrogate indicators, and historical precedents, etc). Furthermore, there are no clear rules outlining which health gains are preferred. Rocchi et al have called for the development of a structured weighting system for evidence and values for reimbursement decision-making. ${ }^{26}$ In this study, authors have concluded that the main way to demonstrate consistent transparency is to be able to explain how seemingly different decisions are reached by different groups or at different times. ${ }^{26}$

\section{HTA research agenda for orphan drugs}

Evidently, there are many problems in the process of reimbursement decision-making for orphan drugs. The emphasis on one single appraisal criterion is not the answer, as it would marginalize all the other equally important criteria. Appraisal decisions strongly depend on the point of view of health authorities. Last but not least, the lack of quality evidence raises the possibility for inconsistent and inadequate access to orphan drugs. A potential solution is to make orphan drugs subject to a post-marketing system that would reduce this evidence gap. The European Pharmaceutical Forum has called for early dialogue between orphan drug stakeholders in order to reconcile the need for treatment access to the cost-effectiveness and safety concerns. ${ }^{2}$ Regulators are already applying a number of different ways to deal with these issues, such as conditional listing, drug restrictions, and risk sharing schemes.
Despite the fact that access to orphan drugs is within the power of each member state, the European Commission is looking very closely at this issue and has been consistently calling for more cooperation and coordination between market authorization authorities in the face of EMA and reimbursement decision-makers at a national level. Following a study on the feasibility of creating a mechanism for the exchange of knowledge on the clinical added value for orphan medicinal products (CAVOMP), the EU Committee of Experts on Rare Diseases (EUCERD) was asked to make recommendations on potential ways to facilitate scientific information exchange on orphan medicinal products in order to support the member states in their processes of making informed decisions on the scientific assessment of the clinical effectiveness of an orphan medicinal product. EUCERD's recommendation has highlighted the fact that the life cycle of an orphan medicinal product is a continuum of evidence generation which is necessary to assessors and decision makers, as well as being important to improve the good use of medicines. ${ }^{1}$

EUCERD conception consists of the following four time points of orphan drug information flow: ${ }^{1} 1$ ) early dialogue between the sponsor, EMA, and HTA bodies leading to a common understanding of data available at marketing authorization and data possibly available post-authorization; 2) information exchange (evidence generation plan with the objective that post-marketing authorization studies are thoroughly defined and relevant, and that the overall evidence generation plan is truly aimed at building understanding of the role of the medicinal product in the therapeutic strategy); 3) follow-up of the evidence generation plan; and 4) updated core HTA information for the assessment of (relative) effectiveness. Special attention is given to the fact that the different actions at the different time points can be implemented as soon as they become possible, rather than waiting for the entire process to be established. ${ }^{1}$

In addition to CAVOMP, there are a number of ongoing European activities in this area, such as the Mechanism for Coordinated Access to orphan medical products (MoCA) within the Process on Corporate Responsibility in the Field of Pharmaceuticals - Platform on Access to Medicines in Europe, initiated by the European Commission. ${ }^{28}$ The purpose of MOCA is pretty much same: to identify common tools to assess the added value of orphan drugs.

The importance of real world quality evidence is obvious for orphan drugs. Also, decision makers would like to rely on multiple sources of integrated data. They would like to see more clarity on orphan drugs' relative effectiveness, 
secure outcomes (on morbidity, mortality, quality of life, etc), decent economic evaluations, and prognostic models. Epidemiological registries are emerging as potential gathering points for global cooperation in the field of rare diseases. They are a combined tool for understanding the natural history of a disease, as well as for monitoring and evaluating safety and cost-effectiveness. There is a huge need for multidisciplinary multi-stakeholder partnerships to reduce the overall level of uncertainty that hinder finding sustainable solutions for orphan drug access.

\section{Conclusion}

The balance between the value of evidence of a health technology and the access to it is a central issue for today's public health. Reimbursement decision-making for orphan drugs is a debate of policy priorities, health system specifics, and societal attitudes. Clear, transparent, and consistent reimbursement decision-making criteria are a legitimate key for securing sustainability and maximizing welfare. This is why it is imperative to create a decision-making framework that would be capable of formally detecting and quantifying all the values that mirror the impact of orphan drugs to patients, society, and payers. Health authorities need to pursue a multidisciplinary analysis on a range of criteria, ensuring an explicit understanding of the trade-offs for decisions on eligibility of reimbursement. The lack of transparency and relevance in the assessment and appraisal of orphan drug value results in irrational and conflicted decisions, which do not promote the wise use of health care resources and do not allow for equal treatment of rare disease patients.

Rarity means that the quality of orphan drug evidence is not the same as for conventional therapies. Closing this gap is another crucial point for the timely access to these products. Rational reimbursement decisions can only be achieved if real world data are available. The only reasonable way to accept a higher valuation of orphan drug benefits is if these are demonstrated empirically. The generation of evidence goes far beyond pre-market authorization trials and requires transnational cooperation and coordination. In order to gain such information, stakeholders need to be more flexible and to initiate partnerships to evaluate the added value of orphan drugs. Approaches, such as conditional listing, drug restrictions, risk sharing schemes, and epidemiological registries, may be useful to gather a critical amount of experience that would rationalize decision-making. Early constructive dialogue among orphan drug stakeholders and elaboration of orphan drug-tailored methodology tools could set the scene for ongoing accumulation of evidence, as well as for proper and timely assessment and appraisal.

\section{Disclosure}

The authors report no conflicts of interest in this work.

\section{References}

1. EUCERD recommendation for a CAVOMP information flow [webpage on the Internet]. Luxembourg: European Union Committee of Experts on Rare Diseases (EUCERD); 2012. Available from: http://www.eucerd. eu/?post_type=document\&p=1446. Accessed November 2, 2013.

2. European Union High Level Pharmaceutical Forum 2005-2008. Final conclusions and recommendations. Brussels: European Commission; 2008. Available from: http://ec.europa.eu/enterprise/sectors/healthcare/ files/docs/pharmaforum_final_conclusions_en.pdf. Accessed November 2, 2013.

3. Dupont AG, Van Wilder PB. Access to orphan drugs despite poor quality of clinical evidence. Br J Clin Pharmacol. 2011;71(4):488-496.

4. Iskrov G, Miteva-Katrandzhieva T, Stefanov R. Challenges to orphan drugs access in Eastern Europe: the case of Bulgaria. Health Policy. 2012;108(1):10-18.

5. Drummond M, Evans B, LeLorier J, et al. Evidence and values: requirements for public reimbursement of drugs for rare diseases - a case study in oncology. Can J Clin Pharmacol. 2009;16(2):e273-e281; discussion e282-e284.

6. Trask LS. Chapter 1. Pharmacoeconomics: principles, methods, and applications. In: Wells BG, editor. Pharmacotherapy: A Pathophysiologic Approach. 8th ed. New York: McGraw-Hill; 2011.

7. Miyamoto BE, Kakkis ED. The potential investment impact of improved access to accelerated approval on the development of treatments for low prevalence rare diseases. Orphanet J Rare Dis. 2011;6:49.

8. Le Jeunne C. [Assessment of actual benefits of new drugs by the Transparency Committee]. J Fr Ophtalmol. 2008;31(1):90-93. French.

9. Drummond M, Brixner D, Gold M, Kind P, McGuire A, Nord E; Consensus Development Group. Toward a consensus on the QALY. Value Health. 2009;12 Suppl 1:S31-S35.

10. Schlander M. The use of cost-effectiveness by the National Institute for Health and Clinical Excellence (NICE): no(t yet an) exemplar of a deliberative process. $J$ Med Ethics. 2008;34(7):534-539.

11. Weinstein MC. The cost-effectiveness of orphan drugs. Am J Public Health. 1991;81(4):414-415.

12. Drummond MF, Wilson DA, Kanavos P, Ubel P, Rovira J. Assessing the economic challenges posed by orphan drugs. Int J Technol Assess Health Care. 2007;23(1):36-42.

13. Eichler HG, Kong SX, Gerth WC, Mavros P, Jönsson B. Use of cost-effectiveness analysis in health-care resource allocation decisionmaking: how are cost-effectiveness thresholds expected to emerge? Value Health. 2004;7(5):518-528.

14. McCabe C, Claxton K, Culyer AJ. The NICE cost-effectiveness threshold: what it is and what that means. Pharmacoeconomics. 2008;26(9):733-744.

15. Drummond MF. Challenges in the economic evaluation of orphan drugs. Eurohealth. 2008;14(2):16-17.

16. Mauskopf JA, Sullivan SD, Annemans L, et al. Principles of good practice for budget impact analysis: report of the ISPOR Task Force on good research practices - budget impact analysis. Value Health. 2007;10(5):336-347.

17. Niezen MG, de Bont A, Busschbach JJ, Cohen JP, Stolk EA. Finding legitimacy for the role of budget impact in drug reimbursement decisions. Int J Technol Assess Health Care. 2009;25(1):49-55.

18. Mauskopf J, Chirila C, Birt J, Boye KS, Bowman L. Drug reimbursement recommendations by the National Institute for Health and Clinical Excellence: have they impacted the National Health Service budget? Health Policy. 2013;110(1):49-59. 
19. Mauskopf J, Chirila C, Masaquel C, et al. Relationship between financial impact and coverage of drugs in Australia. Int J Technol Assess Health Care. 2013;29(1):92-100.

20. Denis A, Mergaert L, Fostier C, Cleemput I, Simoens S. Budget impact analysis of orphan drugs in Belgium: estimates from 2008 to 2013. J Med Econ. 2010;13(2):295-301.

21. De Varax A, Letellier M, Börtlein G; for Alcimed. Study on Orphan Drugs, 2004. Paris; Alcimed; 2004. Available from: http://ec.europa. eu/health/files/orphanmp/doc/pricestudy/final_final_report_part_1_ web_en.pdf. Accessed November 2, 2013.

22. Schey C, Milanova T, Hutchings A. Estimating the budget impact of orphan medicines in Europe: 2010-2020. Orphanet J Rare Dis. 2011;6:62.

23. Rubinstein YR, Groft SC, Bartek R, et al. Creating a global rare disease patient registry linked to a rare diseases biorepository database: Rare Disease-HUB (RD-HUB). Contemp Clin Trials. 2010;31(5):394-404.
24. Rosenberg-Yunger ZR, Daar AS, Thorsteinsdóttir H, Martin DK. Priority setting for orphan drugs: an international comparison. Health Policy. 2011;100(1):25-34.

25. Baltussen R, Niessen L. Priority setting of health interventions: the need for multi-criteria decision analysis. Cost Eff Resour Alloc. 2006;4:14.

26. Rocchi A, Menon D, Verma S, Miller E. The role of economic evidence in Canadian oncology reimbursement decision-making: to lambda and beyond. Value Health. 2008;11(4):771-783.

27. Koopmanschap MA, Stolk EA, Koolman X. Dear policy maker: have you made up your mind? A discrete choice experiment among policy makers and other health professionals. Int JTechnol Assess Health Care. 2010;26(2):198-204.

28. Final report of the Working group on Mechanism of Coordinated Access to orphan medicinal products. Process on Corporate Responsibility in the field of Pharmaceuticals. Brussels: European Commission; 2013. Available from: http://ec.europa.eu/enterprise/sectors/healthcare/files/ docs/orphans_report_en.pdf. Accessed November 2, 2013.
Orphan Drugs: Research and Reviews

\section{Publish your work in this journal}

Orphan Drugs: Research and Reviews is an international, peer-reviewed, open access journal publishing original research, reports, reviews and commentaries on all areas of the design and development of orphan drugs for the treatment of rare diseases through to clinical applications Clinical outcomes, patient safety, and programs for the development and

\section{Dovepress}

effective, safe, and sustained use of medicines will be a feature of the journal. The manuscript management system is completely online and includes a very quick and fair peer-review system, which is all easy to use. Visit http://www.dovepress.com/testimonials.php to read real quotes from published authors.

Submit your manuscript here: http://www.dovepress.com/orphan-drugs-research-and-reviews-journal 